\title{
Autoradiographic localization of [3H]-Nisoxetine binding sites in the CNS of male and female Japanese quail
}

\author{
Shannon E. Eaton ${ }^{1 *} \mathbb{D}$, James R. Pauly ${ }^{2}$, Deann M. Hopkins ${ }^{2}$ and Chana K. Akins ${ }^{1}$
}

\begin{abstract}
Background: In the central nervous system of mammals, transporters localized on the presynaptic nerve terminals regulate the reuptake of neurotransmitters. These transporters are selective for a specific neurotransmitter such as dopamine (DA) and norepinephrine (NE). Specifically in the synapse, the dopamine transporter (DAT) reuptakes DA and the norepinephrine transporter (NET) reuptakes NE. However previous research has found that avian species do not have a gene for DAT, and therefore, birds may be using the NET to clear both NE and DA from the synapse. The current study aimed to extend this finding by localizing NET expression in male and female Japanese quail (Coturnix japonica) brains using [3H]Nisoxetine, a selective NET blocker.

Results: High densities of binding sites were observed in the olfactory tubercle (OTu), the medial striatum (MSt), and the lateral striatum (LSt). Lower densities of binding sites were detected in the amygdala (AMY) and hypothalamus (Hyp), and low binding was found in the medial preoptic area (MPOA) and the pallium.

Conclusion: The areas with the highest densities of NET are also areas that previous research has shown to have high levels of DA activity but low levels of NE innervation (e.g. striatum). The distribution of this reuptake transporter is consistent with the theory that NET acts to clear both DA and NE from the synapse.
\end{abstract}

Keywords: Norepinephrine transporter, Autoradiography, Dopamine reuptake, Japanese quail

\section{Background}

It has long been accepted that the neurotransmitter dopamine (DA) plays a modulatory role in a variety of motivated behaviors such as feeding, reproductive behavior, and defensive behaviors (Martin \& Myers, 1976; Ottinger \& Balthazart, 1987; Blackburn et al., 1992). Relatively more recent studies have focused on the role of DA in learned reward-directed behaviors for food, sex, and drugs (i.e., Gómez-A et al., 2020; Guadarrama-Bazante and Rodriquez-Manzo, 2019; Volkow et al., 2002). These studies suggest that dopamine's functionality appears to be evident in all vertebrates, including birds and reptiles. Evidence for dopamine's involvement in reward-related

\footnotetext{
*Correspondence: shannon.e.eaton@ASU.edu

1 Department of Psychology, University of Kentucky, Lexington, KY, USA Full list of author information is available at the end of the article
}

behaviors in birds comes from numerous studies on sexual behavior and the use of dopamine agonists and antagonists in male Japanese quail. For example, Balthazart et al. (2002) found that administration of dopamine agonists and antagonists profoundly influenced male sexual behavior either directly or in a modulatory fashion. Akins and her colleagues found that cocaine, a DA agonist, facilitated Pavlovian sexual conditioning and increased resistance to extinguish sexual conditioning (Akins et al., 2017; Levens \& Akins, 2004). Cocaine has also been found to dose dependently elicit a conditioned place preference (Levens \& Akins, 2001) that can be blocked by a DA antagonist (Akins et al., 2004). Thus, the role of DA in evoking reward-directed behaviors appears to be conserved in birds.

While there is significant evidence that dopamine's functionality during reward-related events is similar in 
birds as in mammals (e.g., Akins \& Geary, 2008; Akins et al., 2004; Levens \& Akins, 2001), dopamine's availability and re-uptake in the synapse of the bird brain may differ from that of the mammalian brain. Lovell et al. (2015) investigated the evolution and brain expression of the dopamine transporter (DAT) gene in birds and reptiles. Using a combination of sequence alignments and synteny analysis, they found an absence of the DAT gene in birds (i.e., Zebra finch) and lizards (i.e., Green Anole). Further, their in situ hybridization technique indicated that the norepinephrine transporter (NET) was expressed in the nuclei of birds and reptiles where it was co-localized with DA cell markers. Lovell et al. (2015) suggested that this modified NET expression may serve to functionally compensate for the loss of DAT in birds and reptiles. This is in contrast to mammals in which NET and DAT appear to be segregated in distinct brain nuclei and cell types.

The purpose of the current study was to conduct autoradiography on the brain slices of Japanese quail to localize and determine the density of NETusing [3H]Nisoxetine, a potent and selective NET inhibitor. Male and female quail brains were analyzed to determine whether sex differences were present in NET localization and/or density. The brain areas of interest were the medial and lateral striatum (MSt and LSt, respectively), olfactory tubercle (OTu), hypothalamus (Hyp), amygdala (AMY), and the medial preoptic area (mPOA). These regions of the brain were selected for analysis because they were previously investigated for DA content and expression of DA receptors in the quail brain by Ball and his colleagues (Ball et al., 1995; Cornil \& Ball, 2008; Kleitz et al., 2009). Our predictions about localization and density of NE transporters in these regions of the brain were based on Ball et al. (1995). Also, based on Ball et al. (1995), we predicted no sex differences in NE transporters density between male and female quail in any region.

\section{Methods \\ Animals}

Male $(N=7)$ and female $(N=7)$ Japanese quail (Coturnix japonica) were hatched from eggs (Northwest Gamebirds, Kennewick, WA), raised at the University of Kentucky, and used in the current experiment. All quail were individually housed on a 16:8 L:D cycle, had ad lib access to food and water, and had no environmental enrichment. Quail were cared for according to the National Institutes of Health Guide for the Care and Use of Laboratory Animals. Experimental procedures were approved by the Institutional Animal Care and Use Committee, and animal care was provided by the Division of Laboratory Animal Resources at the University of Kentucky.

\section{Autoradiography}

On post-hatch day 42 , quail were rapidly decapitated and their brains were extracted and immediately frozen using isopentane that had been chilled on a bed of dry ice. Frozen brains were stored at $-80{ }^{\circ} \mathrm{C}$ until sectioning. A Leica CM1850 cryostat (Leica, Nussloch, Germany) was used to make $16 \mu \mathrm{m}$ coronal sections that were thaw-mounted onto Superfrost Plus ${ }^{\circledR}$ slides (Fisher Scientific, Pittsburgh, PA, USA). After sectioning, slides were stored under desiccation at $4{ }^{\circ} \mathrm{C}$ overnight and then stored at $-80^{\circ} \mathrm{C}$.

NET autoradiography was performed using [3H]Nisoxetine, as previously described (Tejani-Butt et al., 1990) with minor modifications. Sections were thawed in a desiccator overnight at $4{ }^{\circ} \mathrm{C}$ before being brought to room temperature. The sections were pre-incubated in Tris- $\mathrm{HCl}$ buffer ( $\mathrm{pH}$ 7.4) containing Tris-HCl $(50 \mathrm{mM}), \mathrm{NaCl}(300 \mathrm{mM}), \mathrm{KCl}(5 \mathrm{mM})$, for $10 \mathrm{~min}$ at $4{ }^{\circ} \mathrm{C}$ before being placed in fresh Tris- $\mathrm{HCl}$ buffer with the addition of $1 \mathrm{nM}[3 \mathrm{H}]$-Nisoxetine for $4 \mathrm{~h}$ at $4{ }^{\circ} \mathrm{C}$. After incubation, the sections were washed two times in Tris- $\mathrm{HCl}$ buffer for 5 min per wash followed by a 5 min wash in deionized water. The sections were then allowed to dry overnight. After drying, the sections were exposed to tritium sensitive hyperfilm and stored in $x$-ray cassettes for six weeks. The films were then developed, imaged, and analyzed.

After the films were developed, images were digitized using a Macintosh-based image analysis system that included a Sony XC-77 CCD camera and a Scion LG-3 frame-grabber. All images were then analyzed by a blinded experimenter using ImageJ (version 1.52, NIH, Bethesda MD, USA). Images on films that were blurry were excluded from the analyses. Binding is reported as uncalibrated optical density and NET binding in regions was quantified on 3 adjacent tissue sections.

\section{Statistical analysis}

All analyses were performed using IBM SPSS Statistics for Windows, version 25 (IBM Corp., Armonk, N.Y., USA). A $2 \times 7$ (sex $\times$ brain area) repeated measures ANOVA was used to compare NET densities across brain areas. The brain areas analyzed were OTu, MSt, LSt, Hyp, AMY, mPOA, and pallium. A GreenhouseGeisser statistic was used to adjust the degrees of freedom (rounded to the nearest whole number) when the assumption of sphericity was violated. Bonferroni post hoc tests were used $(p<0.05)$ when appropriate. Additionally, Student's t-tests were used to analyze differences in NET density between male and female quail in brain areas of interest. 


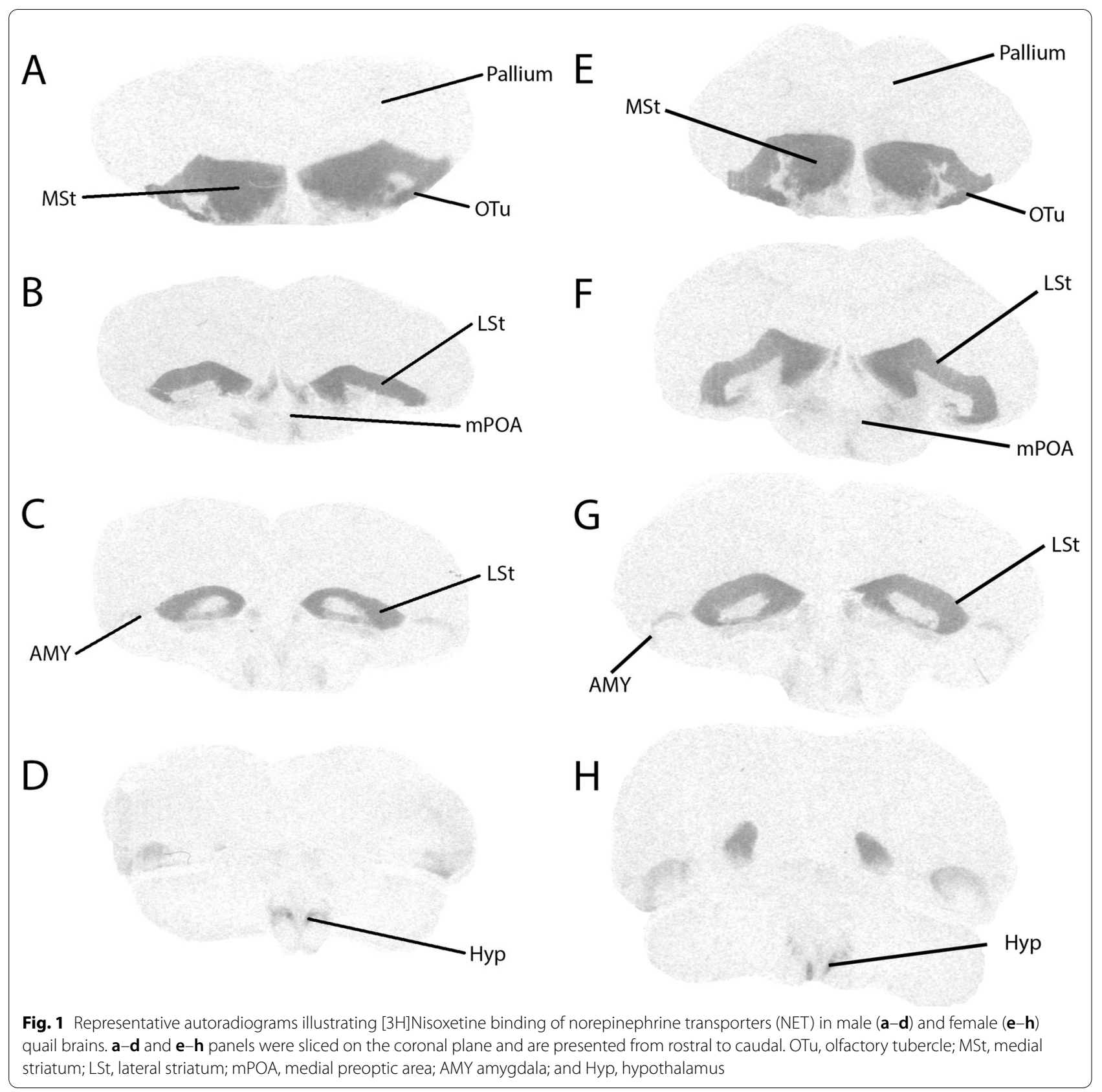

\section{Results}

Figure 1 depicts the quail brain autoradiograms illustrating the density of NET in the targeted brain areas from rostral to caudal. In general, the density of NET was high in the OTu, MSt, and LSt, moderate in the AMY and Hyp, and low in the mPOA and throughout the pallium.

The autoradiograms were analyzed with an RM ANOVA with sex as the independent factor and brain area as the repeated factor. According to the analysis (Fig. 2), there was no overall sex difference in
$[3 \mathrm{H}]$-Nisoxetine binding $[F(1,12)=4.085, p=0.066$, $\left.\eta^{2}=0.215\right]$, and there was no interaction between sex and brain area $\left[F(3,33)=1.508, p=0.79, \eta^{2}=0.173\right]$. However, there was an overall significant main effect of brain area $\left[F(3,33)=297.523, p<0.001, \eta^{2}=0.961\right]$. Bonferroni post hoc tests revealed that the OTu, MSt, and LSt had a greater density of $[3 \mathrm{H}]$-Nisoxetine binding than the AMY, mPOA, Hyp, and pallium $(p<0.05)$ but were not significantly different from one another. Additionally, more binding was evident in the Hyp than in the AMY, mPOA, and pallium $(p<0.05)$, and the AMY had more 


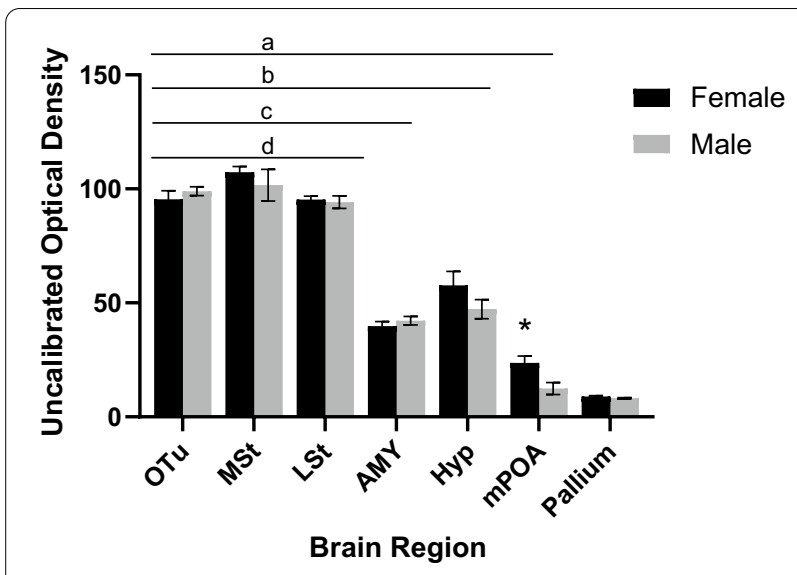

Fig. 2 [3H]Nisoxetine binding in various brain regions of male and female Japanese quail. Each bar and brackets represent the mean of the region $\pm S E M$. a indicates a significant difference from the Pallium, $p<.05$. $b$ indicates a significant difference from mPOA, $p<.05$. $c$ indicates a significant difference from Hyp, $p<.05$. d indicates a significant difference from AMY, $p<.05 .{ }^{*}$ indicates a significant sex difference in the MPOA as indicated by ${ }^{*}, p<.05$. OTu, olfactory tubercle; MSt, medial striatum; LSt, lateral striatum; mPOA, medial preoptic area; AMY amygdala; and Hyp, hypothalamus

binding than the mPOA and pallium. The pallium had less binding than all other areas $(p<0.05)$.

Similar to Ball et al. (1995), we analyzed sex differences in each brain area with Student's t-tests to better identify sex differences that might only be evident within a particular brain area. The t-tests revealed a significant sex difference in the mPOA $[t(12)=4.451, p=0.001]$. The mPOA of females $(M=26.31, \mathrm{SEM}=1.67)$ had more $[3 \mathrm{H}]$ nisoxetine binding than the mPOA of males $(M=12.4666 \mathrm{SEM}=2.62)$. No other sex differences were evident: MSt $[t(12)=0.947, p=0.362]$; OTu $[t(12)=1.297 ., p=0.219$.]; LSt $[t(12)=0.446, p=0.663]$; AMY $[t(12)=0.855, p=0.409] ; \quad$ Hyp $[t(12)=1.63$, $p=0.129]$; and pallium $[t(12)=1.293, p=0.220]$.

\section{Discussion}

The purpose of the current study was to localize and determine the relative density of NET in the brain areas of male and female quail. In the current study, autoradiograms revealed NET throughout the quail brain with transporters most densely located in the OTu, MSt, and LSt, moderately dense in the AMY and Hyp, and least dense in the mPOA and throughout the pallium. The areas where we found the highest $[3 \mathrm{H}]$ nisoxetine binding are the same areas where a high density of dopaminergic neurons and DA receptors have previously been found in the quail brain (Ball et al., 1995; Cornil \& Ball, 2008; Kleitz et al., 2009). Therefore, the high levels of binding in these areas may be evidence for the role of NET in clearing DA from the synapse in brain areas associated with motivated behaviors.

Although the current study did not directly analyze DA and NE differences in the regions analyzed, DA and NE innervation have been examined previously in both birds and mammals. For example, the MSt and LSt in quail are thought to be close analogs to the striatum and nucleus accumbens in mammals (Kleitz et al., 2009; Reiner et al., 2004). Previous research has shown that the striatum is more heavily innervated by DA compared to NE in both birds (Cornil \& Ball, 2008; Smith et al., 2015) and mammals (Del Pino et al., 2011; Jacobowitz \& Richardson, 1978). In the current study, NET binding was high in the striatum of the quail brain. Therefore, it is possible that the high density of NET found in the striatum of the quail brain may be the main DA transporter in this region.

Striatal dopaminergic innervation is associated with motivated and reward related behaviors including addiction related behaviors in birds and mammals. In mice, it appears that DAT is required for drug-induced behavioral sensitization (for review see, Uhl et al., 2002). Unlike DAT knockout mice that do not develop cocaine-induced behavioral sensitization (Mead et al., 2002), quail do acquire a cocaine-induced behavioral sensitization following repeated administration of cocaine (Gill et al., 2015). Cocaine acts to block reuptake at all monoaminergic (i.e., DA, NE, and serotonin) transporter sites. Therefore, the expression of cocaine-induced sensitization in quail may be a result of cocaine blocking the activity of NET (i.e., blocking the reuptake of DA). Although, it appears that quail do not have a gene for DAT (Lovell et al., 2015), the current study found high levels of NET binding in the striatum. It, therefore, may follow that these behaviors usually associated with DAT are more likely to be associated with NET in birds. Taken together, the claim by Lovell et al. (2015) that NET may be responsible for clearing DA from the synapse in aves, appears to be supported by the current results.

The current study found evidence for moderate [3H] nisoxetine binding in the AMY and the Hyp. One possible explanation for this finding is that NET may be acting as both an adrenoceptive and dopaminoceptive transporter in these regions. In rodents, even though both DA and NE are found in the AMY, NE is found at consistent low levels throughout the AMY whereas DA is primarily localized to anterior and basolateral AMY at a much greater concentration compared to NE (BenAri et al., 1975; Kilts \& Anderson, 1986). Despite localized DA innervation in the AMY, there are relatively few DA receptors in the AMY (Charuchinda et al., 1987). Our results are consistent with previous research that suggests cross-talk between dopaminergic and adrenergic systems. Specifically, Yamamoto et al. (2007) found that 
DA directly acts on adrenergic receptors in the lateral AMY. Similar to the AMY of rodents, the AMY of birds is innervated by both dopaminergic and adrenergic neurons. Specifically, birds have a greater ratio of DA to NE in the AMY (Divac et al., 1985), but they also have few DA receptors (Ball et al., 1995) and high concentrations of NE receptors (Balthazart et al., 1989). However, there is no direct evidence for cross-talk in the AMY of birds. Similar $[3 \mathrm{H}]$ nisoxetine binding in AMY, the current study found a moderate level of $[3 \mathrm{H}]$ nisoxetine binding in the Hyp of quail. Previous research indicates that DA is readily found throughout the Hyp, whereas NE tends to be more localized to the ventromedial nucleus and the mPOA (Palkovits et al., 1974). Similar to the AMY, even though DA is found throughout the hypothalamus, there are relatively few DA receptors found in this area. Additionally, like in the AMY of rodents, DA binds to noradrenergic receptors in the mPOA (Cornil et al., 2002) and modulates sexual behavior in quail (Cornil et al., 2005). Therefore, it appears that NET densities in the AMY and Hyp may be related to levels of both dopaminergic and/or noradrenergic innervation, specifically areas with greater DA and NE innervation have higher NET densities.

Surprisingly, the current study found that female quail had a greater density of NET in the MPOA than males. Previous research in rodents indicates that the preoptic area (POA) is larger and more densely packed with cells in females compared to males (Bleier et al., 1982). However, even with a larger POA, sex differences in rats have not been found in DA (Carlsson \& Carlsson, 1998) nor in NE content (Reznikov \& Nosenko, 1995). Similar to rodents, sex differences in DA levels in the POA have not been found in quail (Ottinger et al., 1986; Balthazart et al,. 1992) nor in DA receptor density in the POA (Ball et al., 1995). However, previous research has consistently found that female quail have more NE in the POA than males (Balthazart et al., 1992; Ottinger et al., 1986). Furthermore, the area of noradrenergic innervation in the POA is larger in females than in males (Bailhache \& Balthazart, 1993). Taken together, previous research suggests that perhaps the sex difference observed in the current study may be more likely due to higher NE levels in the $\mathrm{MPOA}$ in female quail compared to male quail rather than as it relates to DA innervation in the mPOA.

\section{Conclusions}

In summary, the current study is, to date, the first to localize and determine the density of NET in quail. The main limitation of the current study is that we did not co-localize NET with dopaminergic markers. When examined against previous literature, we found a higher density of NET in areas known to have high dopaminergic activity, and lower densities in areas known to have less DA and NE innervation. The findings provide further support for previous research (Lovell et al., 2015) that suggests that NET expression may compensate for a loss of DAT in birds and other sauropsids.

\section{Abbreviations}

AMY: Amygdala; DA: Dopamine; DAT: Dopamine transporter; Hyp: Hypothalamus; LSt: Lateral striatum; mPOA: Medial preoptic area; MSt: Medial striatum; NE: Norepinephrine; NET: Norepinephrine transporter; OTu: Olfactory tubercle; POA: Preoptic area.

\section{Acknowledgements \\ National Institute on Alcohol Abuse and Alcoholism training grant T32 AA027488 awarded to SEE.}

\section{Authors' contributions}

All authors contributed to the study conception and design. Material preparation and data collection were performed by SEE and DMH in the lab of JRP. Data analyses were performed by SEE. The first draft of the manuscript was written by SEE and CKA and all authors commented on previous versions of the manuscript. All authors read and approved the final manuscript.

\section{Funding}

National Institute on Alcohol Abuse and Alcoholism training grant T32 AA027488 awarded to SEE and provided training and a stipend to SEE.

\section{Availability of data and materials}

The datasets generated during and/or analyzed during the current study are available from the corresponding author (SEE) on reasonable request.

\section{Declarations}

Ethics approval and consent for participation

Approved by university of Kentucky's IACUC committee, reference number: $\mathrm{N} / \mathrm{A}$.

Consent for publication

N/A.

Competing interests

The authors declare that they have no competing interests.

\section{Author details}

${ }^{1}$ Department of Psychology, University of Kentucky, Lexington, KY, USA.

${ }^{2}$ Department of Pharmaceutical Sciences, College of Pharmacy, University of Kentucky, Lexington, KY, USA.

Received: 11 December 2020 Accepted: 1 September 2021

Published online: 22 September 2021

\section{References}

Akins, C. K., Bolin, B. L., \& Gill, K. E. (2017). Cocaine preexposure enhances sexual conditioning and increases resistance to extinction in male Japanese quail. Learning \& Behavior, 45(3), 313-322.

Akins, C. K., \& Geary, E. H. (2008). Cocaine-induced behavioral sensitization and conditioning in male Japanese quail. Pharmacology Biochemistry and Behavior, 88(4), 432-437.

Akins, C. K., Levens, N., Prather, R., Cooper, B., \& Fritz, T. (2004). Dose-dependent cocaine place conditioning and D1 dopamine antagonist effects in male Japanese quail. Physiology \& Behavior, 82(2-3), 309-315.

Bailhache, T., \& Balthazart, J. (1993). The catecholaminergic system of the quail brain: Immunocytochemical studies of dopamine $\beta$-hydroxylase and tyrosine hydroxylase. Journal of Comparative Neurology, 329(2), 230-256. 
Ball, G. F., Casto, J. M., \& Balthazart, J. (1995). Autoradiographic localization of Dl-like dopamine receptors in the forebrain of male and female Japanese quail and their relationship with immunoreactive tyrosine hydroxylase. Journal of Chemical Neuroanatomy, 9(2), 121-133.

Balthazart, J., Baillien, M., \& Ball, G. F. (2002). Interactions between aromatase (estrogen synthase) and dopamine in the control of male sexual behavior in quail. Comparative Biochemistry and Physiology Part b: Biochemistry and Molecular Biology, 132(1), 37-55.

Balthazart, J., Ball, G. F., \& McEwen, B. S. (1989). An autoradiographic study of a 1-adrenergic receptors in the brain of the Japanese quail (Coturnix coturnix japonica). Cell and Tissue Research, 258(3), 563-568.

Balthazart, J., Foidart, A., Sante, P., \& Hendrick, J. C. (1992). Effects of a-methylpara-tyrosine on monoamine levels in the Japanese quail: Sex differences and testosterone effects. Brain Research Bulletin, 28(2), 275-288.

Ben-Ari, Y., Zigmond, R. E., \& Moore, K. E. (1975). Regional distribution of tyrosine hydroxylase, norepinephrine and dopamine within the amygdaloid complex of the rat. Brain Research, 87(1), 96-101.

Blackburn, J. R., Pfaus, J. G., \& Phillips, A. G. (1992). Dopamine functions in appetitive and defensive behaviours. Progress in Neurobiology, 39(3), 247-279.

Bleier, R., Byne, W., \& Siggelkow, I. (1982). Cytoarchitectonic sexual dimorphisms of the medial preoptic and anterior hypothalamic areas in guinea pig, rat, hamster, and mouse. Journal of Comparative Neurology, 212(2), 118-130.

Carlsson, M., \& Carlsson, A. (1998). A regional study of sex differences in rat brain serotonin. Progress in Neuro-Psychopharmacology and Biological Psychiatry, 12(1), 53-61.

Charuchinda, C., Supavilai, P., Karobath, M., \& Palacios, J. M. (1987). Dopamine D2 receptors in the rat brain: Autoradiographic visualization using a highaffinity selective agonist ligand. Journal of Neuroscience, 7(5), 1352-1360.

Cornil, C. A., \& Ball, G. F. (2008). Interplay among catecholamine systems: Dopamine binds to a2-adrenergic receptors in birds and mammals. Journal of Comparative Neurology, 511(5), 610-627.

Cornil, C. A., Balthazart, J., Motte, P., Massotte, L., \& Seutin, V. (2002). Dopamine activates noradrenergic receptors in the preoptic area. Journal of Neuroscience, 22(21), 9320-9330.

Cornil, C. A., Dejace, C., Ball, G. F., \& Balthazart, J. (2005). Dopamine modulates male sexual behavior in Japanese quail in part via actions on noradrenergic receptors. Behavioural Brain Research, 163(1), 42-57.

Del Pino, J., Martínez, M. A., Castellano, V. J., Ramos, E., Martínez-Larrañaga, M. R., \& Anadón, A. (2011). Effects of prenatal and postnatal exposure to amitraz on norepinephrine, serotonin and dopamine levels in brain regions of male and female rats. Toxicology, 287(1-3), 145-152.

Divac, I., Mogensen, J., \& Björklund, A. (1985). The prefrontal 'cortex'in the pigeon. Biochemical Evidence. Brain Research, 332(2), 365-368.

Gill, K. E., Madison, F. N., \& Akins, C. K. (2015). Cocaine-induced sensitization correlates with testosterone in male Japanese quail but not with estradiol in female Japanese quail. Hormones and Behavior, 67, 21-27.

Gómez-A, A., Shnitko, T. A., Caref, K. L., Nicola, S. M., \& Robinson, D. L. (2020). Stimuli predicting high-calorie reward increase dopamine release and drive approach to food in the absence of homeostatic need. Nutritional Neuroscience. https://doi.org/10.1080/1028415X.2020.1782613

Guadarrama-Bazante, I. L., \& Rodríguez-Manzo, G. (2019). Nucleus accumbens dopamine increases sexual motivation in sexually satiated male rats. Psychopharmacology, 236(4), 1303-1312.

Jacobowitz, D. M., \& Richardson, J. S. (1978). Method for the rapid determination of norepinephrine, dopamine, and serotonin in the same brain region. Pharmacology Biochemistry and Behavior, 8(5), 515-519.

Kilts, C. D., \& Anderson, C. M. (1986). The simultaneous quantification of dopamine, norepinephrine and epinephrine in micropunched rat brain nuclei by on-line trace enrichment HPLC with electrochemical detection: Distribution of catecholamines in the limbic system. Neurochemistry International, 9(3), 437-445.

Kleitz, H. K., Cornil, C. A., Balthazart, J., \& Ball, G. F. (2009). Species differences in the relative densities of D1-and D2-like dopamine receptor subtypes in the Japanese quail and rats: An in vitro quantitative receptor autoradiography study. Brain, Behavior and Evolution, 73(2), 81-90.

Levens, N., \& Akins, C. K. (2001). Cocaine induces conditioned place preference and increases locomotor activity in male Japanese quail. Pharmacology Biochemistry and Behavior, 68(1), 71-80.

Levens, N., \& Akins, C. K. (2004). Chronic cocaine pretreatment facilitates Pavlovian sexual conditioning in male Japanese quail. Pharmacology Biochemistry and Behavior, 79(3), 451-457.

Lovell, P. V., Kasimi, B., Carleton, J., Velho, T. A., \& Mello, C. V. (2015). Living without DAT: Loss and compensation of the dopamine transporter gene in sauropsids (birds and reptiles). Scientific Reports, 5(1), 1-12.

Martin, G. E., \& Myers, R. D. (1976). Dopamine efflux from the brain stem of the rat during feeding, drinking, and lever-pressing for food. Pharmacology Biochemistry and Behavior, 4(5), 551-560.

Mead, A. N., Rocha, B. A., Donovan, D. M., \& Katz, J. L. (2002). Intravenous cocaine induced-activity and behavioural sensitization in norepinephrine-, but not dopamine-transporter knockout mice. European Journal of Neuroscience, 16(3), 514-520.

Ottinger, M. A., \& Balthazart, J. (1987). Brain monoamines and sexual behavior in Japanese quail: Effects of castration and steroid replacement therapy. Behavioural Processes, 14(2), 197-216.

Ottinger, M. A., Schumacher, M., Clarke, R. N., Duchala, C. S., Turek, R., \& Balthazart, J. (1986). Comparison of monoamine concentrations in the brains of adult male and female Japanese quail. Poultry Science, 65(7), 1413-1420.

Palkovits, M., Brownstein, M., Saavedra, J. M., \& Axelrod, J. (1974). Norepinephrine and dopamine content of hypothalamic nuclei of the rat. Brain Research, 77(1), 137-149.

Reiner, A., Perkel, D. J., Bruce, L. L., Butler, A. B., Csillag, A., Kuenzel, W., et al. (2004). Revised nomenclature for avian telencephalon and some related brainstem nuclei. Journal of Comparative Neurology, 473(3), 377-414.

Reznikov, A. G., \& Nosenko, N. D. (1995). Catecholamines in steroid-dependent brain development. The Journal of Steroid Biochemistry and Molecular Biology, 53(1-6), 349-353.

Smith, A. R., Garris, P. A., \& Casto, J. M. (2015). Real-time monitoring of electrically evoked catecholamine signals in the songbird striatum using in vivo fast-scan cyclic voltammetry. Journal of Chemical Neuroanatomy, 66, 28-39.

Tejani-Butt, S. M., Brunswick, D. J., \& Frazer, A. (1990). [3H] Nisoxetine: A new radioligand for norepinephrine uptake sites in brain. European Journal of Pharmacology, 191(2), 239-243.

Uhl, G. R., Hall, F. S., \& Sora, I. (2002). Cocaine, reward, movement and monoamine transporters. Molecular Psychiatry, 1, 21-26.

Volkow, N. D., Fowler, J. S., Wang, G. J., \& Goldstein, R. Z. (2002). Role of dopamine, the frontal cortex and memory circuits in drug addiction: insight from imaging studies. Neurobiology of Learning and Memory, 78(3), 610-624.

Yamamoto, R., Ueta, Y., \& Kato, N. (2007). Dopamine induces a slow afterdepolarization in lateral amygdala neurons. Journal of Neurophysiology, 98(2), 984-992.

\section{Publisher's Note}

Springer Nature remains neutral with regard to jurisdictional claims in published maps and institutional affiliations. 